\title{
Groupwise Rigid Registration of Wrist Bones
}

\author{
Martijn van de Giessen ${ }^{1,2,3,4}$, Frans M. Vos ${ }^{1,5}$, \\ Cornelis A. Grimbergen ${ }^{4}$, Lucas J. van Vliet ${ }^{1}$, and Geert J. Streekstra ${ }^{4}$ \\ 1 Quantitative Imaging Group, Delft University of Technology, The Netherlands \\ 2 Division of Image Processing, Leiden University Medical Center, The Netherlands \\ 3 Department of Intelligent Systems, Delft University of Technology, The Netherlands \\ 4 Dept. of Biomed. Engineering and Physics, AMC Amsterdam, The Netherlands \\ 5 Dept. of Radiology, AMC Amsterdam, The Netherlands \\ $\mathrm{m}$. vandegiessen@lumc.nl
}

\begin{abstract}
We present an extension of the symmetric ICP algorithm that is unbiased for an arbitrary number $(N \geq 2)$ of shapes, using rigid transformations and scaling. The method does not require the selection of a reference shape or registration order and hence it is unbiased towards any of the registered shapes. The functional to be minimized is non-linear in the transformation parameters and thus computationally complex. We therefore propose a first order approximation that estimates the transformation parameters in a closed form, with computational complexity $\mathcal{O}\left(N^{2}\right)$.

Using a set of wrist bones, we show that the least-squares minimization and the proposed approximation converge to the same solution. Experiments also show that the proposed algorithms lead to smaller registration errors than algorithms that select a reference shape or register to an evolving mean shape. The low computational cost and trivial parallelization enable the alignment of large numbers of bones.
\end{abstract}

\section{Introduction}

Groupwise registration of multiple shapes (i.e. more than two) is a recurring problem in a wide variety of medical applications. Although much interest has been paid to non-rigid groupwise registration, especially intensity based such as in atlas building, some applications, e.g. the groupwise alignment of bone surfaces in orthopedics would benefit more from an unbiased rigid alignment. Although such an alignment can be obtained using the correspondence obtained with a non-rigid method, non-rigid registration results in general heavily depend on the careful tuning of the regularization parameters.

Many methods for the alignment of multiple shapes select one target to which all other shapes are registered or deformed, e.g. [7. This, however biases the registration result to the selected shape. To minimize this bias, 5] proposed a strategy to select a shape that lies the closest the 'mean' shape, while others, e.g. 32 proposed to evolve a mean shape. The first method still does not completely remove the bias, while the latter methods add an extra layer of complexity, i.e. estimating the correspondence between registered example shapes and the mean shape.

N. Ayache et al. (Eds.): MICCAI 2012, Part II, LNCS 7511, pp. 155-162, 2012.

(C) Springer-Verlag Berlin Heidelberg 2012 

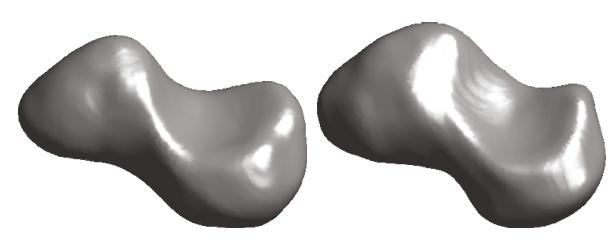

(a)

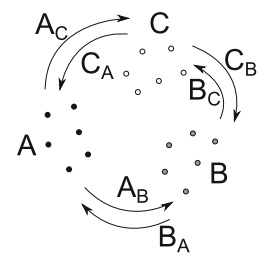

(b)

Fig. 1. (a) Examples of scaphoid bones from three individuals. (b) Three shapes with correspondence relations.

In this paper we propose a new method for the rigid registration (including isotropic scaling) of more than two objects that is inherently unbiased, i.e. it does not depend on the selection of a target shape or the order in which the shapes are processed. Moreover, the algorithm is stable and has a low computational complexity. This method is an extension of the symmetric ICP algorithm [1] to register more than two shapes. The direct extension yields a functional that is nonlinear in its transformation parameters and minimization is computational complex. To amend this we additionally introduce a method that involves separate closed-form estimations of the transformation parameters for each shape. We prove that this method converges to the same minimum as the direct ICP extension. Both algorithm variations are experimentally validated on a large number of subsets of 50 scaphoid bones, a bone in the wrist (See Figure 1a). These experiments demonstrate that the proposed algorithm leads to improved registration results over selecting the 'best' target shape as well as over registering to an evolving mean shape. Moreover, we will also show that the closed-form based variant converges to the same result as the direct extension of the extended ICP algorithm.

\section{Methods}

\subsection{Unbiased ICP Algorithm for $N$ Objects}

We propose to extend the symmetric ICP algorithm to an unbiased algorithm for $N$ shapes. For the sake of clarity we will initially present this algorithm for three shapes $S_{A}, S_{B}$ and $S_{C}$, represented by point clouds. However, it can be directly generalised to $N$ shapes with different representations. We define a set of control points for each shape, denoted by $A, B$ and $C$. For each set of control points, the corresponding points on the other two shapes are determined using the minimum Euclidean distance as the criterion. For example, the corresponding points from $S_{B}$ and $S_{C}$ to $A$ are $B_{A}$ and $C_{A}$. A schematic representation of three shapes and their correspondences is shown in Figure 1b.

The transformation between point sets is found by minimizing the average squared Euclidean distance between corresponding point pairs. We extend the symmetric ICP to include all directional pairwise correspondences: 


$$
\begin{aligned}
J_{n i c p}\left(T_{A}, T_{B}, T_{C}\right)= & \frac{1}{n_{A}}\left\|T_{A}(A)-T_{B}\left(B_{A}\right)\right\|^{2}+\frac{1}{n_{A}}\left\|T_{A}(A)-T_{C}\left(C_{A}\right)\right\|^{2} \\
& +\frac{1}{n_{B}}\left\|T_{B}(B)-T_{A}\left(A_{B}\right)\right\|^{2}+\frac{1}{n_{B}}\left\|T_{B}(B)-T_{C}\left(C_{B}\right)\right\|^{2} \\
& +\frac{1}{n_{C}}\left\|T_{C}(C)-T_{A}\left(A_{C}\right)\right\|^{2}+\frac{1}{n_{C}}\left\|T_{C}(C)-T_{B}\left(B_{C}\right)\right\|^{2}
\end{aligned}
$$

in which $T_{A}, T_{B}$ and $T_{C}$ are transformations from point set $A, B$ or $C$. This cost function is nonlinear in its transformations, since it contains products of the transformation parameters. Therefore, a closed-form solution of the transformations does not exist. The minimization can be done using a nonlinear regression technique, e.g. by the Levenberg-Marquardt algorithm.

Just as in the symmetric ICP algorithm, the minimization of (1) is mathematically ill-posed. The constraint $T_{A}=T_{B}^{-1}$, however, cannot be generalised to more than two transformations. Therefore, we choose the following constraints, that follow a similar rationale:

1. A net scaling of one: $\hat{s}_{A} \hat{s}_{B} \hat{s}_{C}=1$

2. A zero net translation: $\hat{\mathbf{t}}_{A}+\hat{\mathbf{t}}_{B}+\hat{\mathbf{t}}_{C}=\mathbf{0}$

3. A zero net rotation: $\hat{\mathbf{r}}_{A}+\hat{\mathbf{r}}_{B}+\hat{\mathbf{r}}_{C}=\mathbf{0}$

in which $\hat{s}_{A}, \hat{s}_{B}, \hat{s}_{C}$ are the scalings, $\hat{\mathbf{t}}_{A}, \hat{\mathbf{t}}_{B}, \hat{\mathbf{t}}_{C}$ the translations and $\hat{\mathbf{r}}_{A}, \hat{\mathbf{r}}_{B}, \hat{\mathbf{r}}_{C}$ the rotations involved in the transformations $T_{A}, T_{B}, T_{C}$. The rotations are represented by the vectors $\hat{\mathbf{r}}_{A}, \hat{\mathbf{r}}_{B}, \hat{\mathbf{r}}_{C}$ that contain the Rodrigues parameters: the vectors $\hat{\mathbf{r}}_{A}, \hat{\mathbf{r}}_{B}, \hat{\mathbf{r}}_{C}$ are oriented parallel to the rotation axes (also called helical axes) of $A, B$ and $C$ and have a length equal to the magnitude of the rotation angle. These three constraints are included in the nonlinear least squares optimization using Lagrange multipliers.

\subsection{Closed Form Transformation Estimates}

A direct minimization of (1) is computationally expensive, because its non-linear form prevents finding the transformations $T_{A}, T_{B}$ and $T_{C}$ using a closed-form solution. We assume that the concurrent transformation of all clouds can be $a p$ proximated by separate rigid registrations of each cloud that position the clouds at time step $k$ 'in the middle' of all three clouds at time step $k-1$. 'The middle' is then defined as the position and orientation in which the sum of the quadratic Euclidean distances between the points in a cloud and their corresponding points in all clouds (including the transformed cloud at time step $k-1$ ) in the set is minimal. Accordingly, we define the functional $J_{\text {approx }}\left(T_{A}, T_{B}, T_{C}\right)=J_{A}\left(T_{A}\right)+$ $J_{B}\left(T_{B}\right)+J_{C}\left(T_{C}\right)$ with right-hand terms of the form:

$$
\begin{aligned}
J_{A}\left(T_{A}^{(k)}\right)= & \frac{2}{n_{A}}\left\|T_{A}^{(k)}(A)-T_{A}^{(k-1)}(A)\right\|^{2} \\
& +\frac{1}{n_{B}}\left\|T_{A}^{(k)}\left(A_{B}\right)-T_{B}^{(k-1)}(B)\right\|^{2}+\frac{1}{n_{A}}\left\|T_{A}^{(k)}(A)-T_{B}^{(k-1)}\left(B_{A}\right)\right\|^{2} \\
& +\frac{1}{n_{C}}\left\|T_{A}^{(k)}\left(A_{C}\right)-T_{C}^{(k-1)}(C)\right\|^{2}+\frac{1}{n_{A}}\left\|T_{A}^{(k)}(A)-T_{C}^{(k-1)}\left(C_{A}\right)\right\|^{2}(2)
\end{aligned}
$$


It may be observed that each of the functionals $J_{A}\left(T_{A}^{(k)}\right), J_{B}\left(T_{B}^{(k)}\right)$ and $J_{C}\left(T_{C}^{(k)}\right)$ depends on a single transformation, for which a closed-form solution exists. The first term in (2) effectively imposes that all shapes are equally involved. In comparison to (1), one transformation maps one shape unidirectionally onto the other shapes within each functional. The reverse mapping, i.e. mapping the other shapes onto the one, is actually contained in the other functionals.

Enforcing the constraints in Section 2.1 would result in a nonlinear problem. Instead, we opted to add a separate 'normalization' step after $T_{A}, T_{B}$ and $T_{C}$ are all updated.

For $N$ shapes, $N$ functionals as in (2) are needed, each with $\left(\begin{array}{c}N \\ 2\end{array}\right)$ terms.

Normalization of scalings and rotations. After each update of all transformations, the net scaling of all shapes combined is $s_{A} s_{B} s_{C}=\bar{s}$, where $s_{A}, s_{B}, s_{C}$ represent the current scale estimates. We adopt the next normalization to retain the ratio of the estimates:

$$
\hat{s}_{A}=s_{A} / s_{n}, \hat{s}_{B}=s_{B} / s_{n}, \hat{s}_{C}=s_{C} / s_{n}
$$

in which $s_{n}=\sqrt[3]{\bar{s}}$, such that $\hat{s}_{A} \hat{s}_{B} \hat{s}_{C}=1$. The rotations undergo a similar normalization, so that the relative rotations between the three shapes is kept constant, i.e. $\hat{\mathbf{r}}_{A}+\hat{\mathbf{r}}_{B}+\hat{\mathbf{r}}_{C}=\mathbf{0}$. Let the sum of the Rodrigues parameters after minimizing (2) be $\mathbf{r}_{A}+\mathbf{r}_{B}+\mathbf{r}_{C}=\mathbf{r}_{n}$. Then rotations are normalized as follows

$$
\hat{\mathbf{r}}_{A}=\mathbf{r}_{A}-\frac{1}{3} \mathbf{r}_{n}, \hat{\mathbf{r}}_{B}=\mathbf{r}_{B}-\frac{1}{3} \mathbf{r}_{n}, \hat{\mathbf{r}}_{C}=\mathbf{r}_{C}-\frac{1}{3} \mathbf{r}_{n}
$$

If the rotation matrices that describe the updated rotations are $\hat{R}_{A}, \hat{R}_{B}$ and $\hat{R}_{C}$, the translations $\mathbf{t}_{A}, \mathbf{t}_{B}$ and $\mathbf{t}_{C}$ are updated to

$$
\hat{\mathbf{t}}_{A}=\overline{\mathbf{a}}_{T}-\hat{s}_{A} \hat{R}_{A} \overline{\mathbf{a}}_{S}, \hat{\mathbf{t}}_{B}=\overline{\mathbf{b}}_{T}-\hat{s}_{B} \hat{R}_{B} \overline{\mathbf{b}}_{S}, \hat{\mathbf{t}}_{C}=\overline{\mathbf{c}}_{T}-\hat{s}_{C} \hat{R}_{C} \overline{\mathbf{c}}_{S}
$$

where $\overline{\mathbf{a}}_{T}, \overline{\mathbf{b}}_{T}, \overline{\mathbf{c}}_{T}$ and $\overline{\mathbf{a}}_{S}, \overline{\mathbf{b}}_{S}, \overline{\mathbf{c}}_{S}$ are the means of $A_{T}, B_{T}, C_{T}$ and $A_{S}, B_{S}, C_{S}$, respectively. It may be noticed that the value of $J_{\text {approx }}\left(T_{A}, T_{B}, T_{C}\right)$ is modified only due to the scale normalization, i.e. the normalization of rotation and translation parameters has no effect if $s_{n}=1$.

\subsection{Equivalence of Both Solutions}

One may notice that when the algorithm in Section 2.2 has converged, it holds for all transformations that $T_{X}^{(k)}=T_{X}^{(k-1)}$ for all clouds $X \in\{A, B, C\}$. Thus, comparing (11) and (2), it holds at convergence that $J_{\text {approx }}=2 J_{\text {nicp }}$. From this one can see that there is a set of transformations for which the global minimum of $J_{\text {nicp }}$ can be obtained with $J_{\text {approx }}$. Furthermore, the same transformations that lead to a global minimum of $J_{n i c p}$, give a global minimum of $J_{\text {approx }}$ : if the global minimum of $J_{\text {nicp }}$ would not be the global minimum of $J_{\text {approx }}$, then it would be possible to find a set of transformations for which holds $J_{\text {approx }}<2 J_{\text {nicp }}$. The latter is not possible, because for any choice of $T_{X}^{(k)}=T_{X}^{(k-1)}$ it holds that $J_{\text {approx }}=2 J_{\text {nicp }}$. It can be proven that $J_{\text {approx }}$ decreases every iteration similar to the pairwise ICP algorithm [1] and thus converges to a local minimum. 
Table 1. Average values and standard deviations of $J$ for $n=400$ points, normalized to the results of Algorithm 3 and mean Target Registration Errors (mm).

\begin{tabular}{|c|c|c|c|c|c|c|}
\hline$N$ & $J_{1}$ & $J_{2}$ & $J_{4}$ & mTRE $_{1}$ & mTRE $_{2}$ & mTRE $_{4}$ \\
\hline \hline 2 & $1.006(0.004)$ & $1.288(0.233)$ & $1.000(0.001)$ & $1.4(0.4)$ & $1.4(0.4)$ & $1.4(0.4)$ \\
\hline 4 & $1.069(0.046)$ & $1.544(0.108)$ & $1.005(0.006)$ & $1.4(0.3)$ & $1.5(0.3)$ & $1.5(0.3)$ \\
\hline 8 & $1.146(0.037)$ & $1.573(0.079)$ & $1.003(0.006)$ & $1.6(0.3)$ & $1.7(0.2)$ & $1.5(0.2)$ \\
\hline 16 & $1.177(0.024)$ & $1.576(0.041)$ & $1.000(0.000)$ & $1.7(0.3)$ & $2.1(0.3)$ & $1.4(0.1)$ \\
\hline
\end{tabular}

\section{$3 \quad$ Experiments}

The performance of two existing algorithms (1) selecting the optimal mean shape [5] and (2) evolving a mean shape [16] and the proposed algorithms (3) in Section 2.1 and (4) in Section 2.2 are assessed in terms of accuracy and precision, as well as computational cost. These criteria are assessed as a function of the number of shapes, $N$, and the number of sampling points, $n$. CT images of 50 scaphoid wrist bones served as an application (See Figure 1a). Each scaphoid was represented by a point cloud, uniformly sampled on the bone surface. Algorithm 1 selects each of the $N$ shapes as a target, registers all other shapes to this target and selects the result with the smallest remaining registration cost. Algorithm 2 takes one shape as initial mean and registers all shapes to this 'mean'. Subsequently the mean is updated by averaging the coordinates of corresponding points.

\subsection{Accuracy and Precision}

A subset of $N$ bones was selected from the total of 50 and registered by Algorithms 1 to 4 . For each outcome we evaluated $J_{n i c p}$ (1) in order to have comparable measures (effectively the algorithm from Section 2.1] Algorithm 3, serves as the reference standard). The resulting measures are $J_{1}, J_{2}, J_{3}$ and $J_{4}$. Each obtained value was divided by $J_{3}$ for normalization. The experiments were repeated 10 times for different subset selections from the 50 objects and for $N \in\{2,4,8,16\}$ shapes and $n \in\{200,400,600,800\}$ surface points. A maximum of $N=16$ was taken since the reference Algorithm 3 became impractically time consuming for higher values. For the same reason a maximum of $n=400$ points was used to register $N=16$ shapes. Each of the $N$ shapes involved in an experiment served as the target shape in Algorithm 2. The accuracies reported for Algorithm 2 were obtained both by averaging over all target shapes and subsets.

The mean normalized values $\bar{J}_{1, \ldots, 4}$ of $J_{1, \ldots, 4}$ for $n=400$ and their standard deviations are shown in Table $1 . \bar{J}_{4}$ always differs less than $1 \%$ from $\bar{J}_{3}$. As expected, registering to a single target shape $\bar{J}_{1}$, for $N>2$, leads to higher values than $\bar{J}_{3}$. Moreover $\bar{J}_{1}$ increases with increasing $N$. This follows directly from 1 as the registration in Algorithm 1 only has a (relatively decreasing) subset of the minimized terms in 1 The initial registration to a target shape in Algorithm 2 is by definition worse than Algorithm 1 as no selection of a shape closest to the mean is involved. Surprisingly, we found that after the initial 


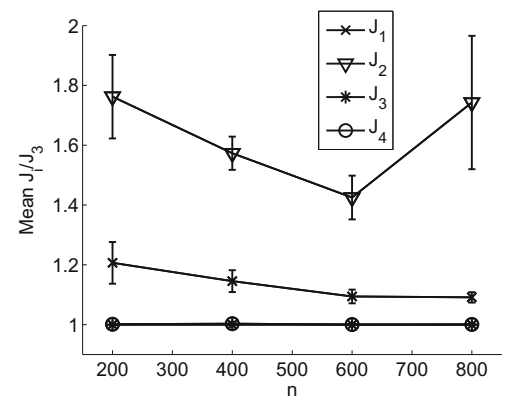

(a)

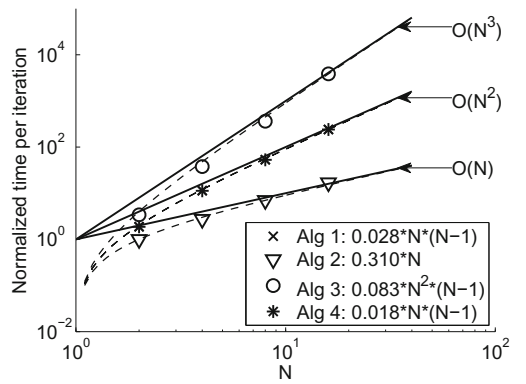

(b)

Fig. 2. (a) Registration results $\bar{J}_{1}, \bar{J}_{2}, \bar{J}_{3}$ and $\bar{J}_{4}$ as a function of the number of sample points for $N=8$ shapes. Error bars denote standard deviations. Results of $\bar{J}_{3}$ and $\bar{J}_{4}$ almost coincide. (b) Normalized average processing times per iteration for Algorithms $1(\times), 2(\nabla), 3(\circ)$ and $4(*)$ as a function of $N$ for $n=400$. The legend shows the functions fitted to the measured times per iteration. Plotted times are normalized to the numbers in the legend. Dashed: normalized theoretical times per iteration. Continuous: order of computational complexity. Results for Algorithm $1(\times)$ and $4(*)$ almost coincide.

registration to a target shape, $\bar{J}_{2}$ increased as the evolving mean was computed. This indicates that the evolving mean differs more from the reference mean than the selected target shape. The large standard deviations for Algorithm 2 are due to variable, non-optimal target shape selections. In all experiments $J_{2}$ was the highest, $J_{1}$ the second highest and $J_{3}$ or $J_{4}$ the lowest. The target shape that gave the lowest value of $J$ in Algorithm 1 typically, but not always, yielded the lowest value of $J$ in Algorithm 2. Registration differences between methods were about 5 degrees with extremes to 15 degrees. An evaluation using non-fiducial corresponding landmarks gave mean Target Registration Errors (mTRE) as in Table 1]. Only for Algorithm 4 was the mTRE constant for increasing $N$.

Figure 2a shows that for an increasing number of points $\bar{J}_{1}$ decreases, while $\bar{J}_{4}$ has a negligible difference from $\bar{J}_{3} \cdot \bar{J}_{2}$ seems to decrease too, for increasing $n$, but makes a sudden jump for $n=800$. Although the standard deviation of $\bar{J}_{2}$ is large for $n=800$, this result was not due to outliers and we attribute these results to convergence to local minima.

\subsection{Computational Complexity}

To assess the computational complexity of Algorithms 1 to 4, the execution time and the numbers of iterations were measured. The algorithms were implemented in MATLAB 7.4.0 on an AMD Opteron Quad Core Processor 250 at $2.0 \mathrm{GHz}$ with 64 GB of memory, using a single core, to assess the computational complexity. The registration times were measered as the times needed until convergence within $1 \%$ of the final outcome to reduce the influence of the stopping criterium. Table 2 collates the mean registration times and their standard deviations for 
Table 2. Average times in seconds needed for convergence of the four registration algorithms for $n=400$. The standard deviations are between parentheses. For Algorithm 2 , both the times for the initial registration to a target shape (I) and the registration to the evolving mean (II) are given.

\begin{tabular}{|c|c|c|c|c|}
\hline$N$ & Reg. 1 & Reg. 2 (I)/(II) & Reg. 3 & Reg. 4 \\
\hline \hline 2 & $1.3(0.4)$ & $0.6(0.2) / 0.6(0.1)$ & $7.0(5.2)$ & $0.8(0.3)$ \\
\hline 4 & $7.13(0.9)$ & $1.8(0.2) / 2.4(0.5)$ & $93.7(30.6)$ & $5.5(1.5)$ \\
\hline 8 & $28.0(4.3)$ & $3.5(0.5) / 7.6(0.5)$ & $1202.5(147.2)$ & $33.8(8.8)$ \\
\hline 16 & $142.1(1.2)$ & $8.9(0.1) / 16.3(3.6)$ & $11560.5(1304.0)$ & $159.4(18.8)$ \\
\hline
\end{tabular}

$n=400$ surface points. Algorithm 2 is clearly the fastest, while Algorithms 1 and 4 take approximately the same time to converge. Algorithm 3 is impractically slow for large numbers of shapes. Running the registration on three cores (to leave one of the core available for the operating system), the registration of 8 and 16 shapes with Algorithm 4 took 11.9 and 55.5 seconds on average, a speedup of approximately 2.85 . This shows that the algorithm can be efficiently parallelized. During the optimization, $J_{4}$ differed less than $1 \%$ from $J_{3}$ at each iteration, which is a strong indication that the computational scheme closely approximates the direct minimization both far from and close to the solution.

To estimate computational complexity as a function of the number of shapes $N$, the functions $a_{1} \cdot N(N-1), a_{2} \cdot N, a_{3} \cdot N^{2}(N-1)$ and $a_{4} \cdot N(N-1)$ are fitted to the processing times per iteration. These fit functions can be derived from identifying the most expensive steps in the four algorithms such as correspondence finding and (in Algorithm 3) estimating the Jacobian. All four fits are shown in the legend of Figure $2 \mathrm{~b}$. Although the processing times per iteration are comparable between Algorithms 1 and 4, Algorithm 4 takes slightly longer for larger numbers of shapes $N$ because the number of iterations of Algorithm 4 increases when $N$ increases. The average processing times per iteration as a function of the number of points $n$ was $\mathcal{O}(n \log n)$ for all four algorithms, corresponding to the complexity of closest point search using a Delaunay triangularization.

\section{Discussion}

We proposed a novel approach to extend the symmetric ICP algorithm to the unbiased registration of $N \geq 3$ shapes. All estimated correspondences between all shapes have equal weight in the cost functions (1) and the registration result does not depend on the order of registration.

Experiments confirmed that the proposed approximate minimization $J_{\text {approx }}$ and a direct minimizationg of $J_{n i c p}$ converge to the same minimum. Assuming Gaussian distributed point correspondences, just as with the symmetric ICP algorithm, the proposed groupwise version gives optimal registration results since (1) is the minimum variance estimator for Gaussian distributed coordinates.

When choosing the best registration to a single target (Algorithm 1), the mean quadratic distance between shapes after registration, assessed by $J_{n i c p}$ (11), were all higher than after a direct minimization of $J_{n i c p}$, and increased with 
the number of shapes $N$. The difference, however, decreased with the number of surface points $n$. Registering to an evolving mean shape (Algorithm 2) resulted in a much higher mean quadratic distance between corresponding points. While direct minimization of $J_{\text {nicp }}$ is practically not feasible for large numbers of shapes due to the order of time complexity $\mathcal{O}\left(N^{3} \times n^{2}\right)$, the proposed approximation has a time complexity of order $\mathcal{O}\left(N^{2} \times n^{2}\right)$. Thus this proposed approximation combines optimal registration accuracy with an acceptable time complexity. Furthermore, the algorithm was shown to be easily parallelizable, allowing an additional reduction registration time.

For clarity of the discussion, the unbiased ICP algorithms in this work estimate corresponding points in the same, separate step as the original ICP algorithm. This can, however, easily be improved, e.g. with a weighted closest point average as in [4], affecting only correspondence estimates such as $B_{A}$ and $C_{A}$.

Using the proposed unbiased ICP algorithm and the approximation presented in this paper, it is possible to perform an unbiased registration with a computational complexity that allows the registration of a large number of bones. The unbiased registration ensures that the estimated bone alignment does not depend on the selection of a target or registration order.

\section{References}

1. Brett, A.D., Taylor, C.J.: A method of automated landmark generation for automated 3D PDM construction. Image and Vision Computing 18(9), 739-748 (2000)

2. Chui, H., Rangarajan, A., Zhang, J., Leonard, C.M.: Unsupervised learning of an atlas from unlabeled point-sets. IEEE Transactions on Pattern Analysis and Machine Intelligence 26(2), 160-172 (2004)

3. Frangi, A.F., Rueckert, D., Duncan, J.S.: Three-dimensional cardiovascular image analysis. IEEE Transactions on Medical Imaging 21(9), 1005-1010 (2002)

4. Granger, S., Pennec, X.: Multi-scale EM-ICP: A Fast and Robust Approach for Surface Registration. In: Heyden, A., Sparr, G., Nielsen, M., Johansen, P. (eds.) ECCV 2002, Part IV. LNCS, vol. 2353, pp. 418-432. Springer, Heidelberg (2002)

5. Park, H., Bland, P.H., Hero III, A.O., Meyer, C.R.: Least Biased Target Selection in Probabilistic Atlas Construction. In: Duncan, J., Gerig, G. (eds.) MICCAI 2005, Part II. LNCS, vol. 3750, pp. 419-426. Springer, Heidelberg (2005)

6. Vos, F.M., de Bruijn, P.W., Aubel, J.G.M., Streekstra, G.J., Maas, M., van Vliet, L.J., Vossepoel, A.M.: A statistical shape model without using landmarks. In: Proceedings 17th International Conference on Pattern Recognition, ICPR17, Cambridge, UK, vol. 3, pp. 714-717. IEEE Computer Society Press (2004)

7. Yang, Y., Bull, A., Rueckert, D., Hill, A.: 3D Statistical Shape Modeling of Long Bones. In: Pluim, J.P.W., Likar, B., Gerritsen, F.A. (eds.) WBIR 2006. LNCS, vol. 4057, pp. 306-314. Springer, Heidelberg (2006) 